\title{
From Elastomers to Thermoplasts - Precise Control of Isotactic Propylene Structure and Properties and the Role of Different Structural Elements in Its Mechanical Behaviour
}

\author{
Maxim A Shcherbina ${ }^{\mathrm{a}, \mathrm{b}}$, Marina Yu. Meshchankina ${ }^{\mathrm{a}}$, \\ Yaroslav I. Odarchenko ${ }^{\mathrm{c}}$, \\ Martin Machat ${ }^{\mathrm{d}}$, Bernhard Rieger ${ }^{\mathrm{d}}$, Sergey N. Chvalun ${ }^{\mathrm{a}, \mathrm{c}}$ \\ a National Research Centre "Kurchatov Institute”, 1 pl. Academika \\ Kurchatova, 123098 Moscow, Russian \\ ${ }^{\mathrm{b}}$ Moscow Institute of Physics and Technology, 4 Institutsky line, \\ 141700 Dolgoprudny, Moscow region, Russian \\ ${ }^{\mathrm{c}}$ Enikolopov Institute of Synthetic Polymer Materials RAS, $70 \mathrm{ul}$. \\ Profsoyuznaya, 117393 Moscow, Russian \\ ${ }^{\mathrm{d}}$ WACKER-Lehrstuhl für Makromolekulare Chemie, Technische \\ Universität München, Lichtenbergstrasse 4, D-85747 Garching bei München, \\ Germany
}

\begin{abstract}
Studies of polypropylene with different degrees of isotacticity have shown a way of the rational design of material with predetermined mechanical properties starting from the synthesis stage already - controlled introducement of stereodefects will allow the smooth adjustment of the Young's modulus and elasticity in the range from plastic to elastomer materials. It was also revealed that modern theoretical models of the elasticity can be successfully applied not only for the description of the mechanical behaviour of polymers, but also for better understanding of the mechanism of elasticity in them. While in the low crystalline materials deformation has Gaussian nature, in the materials of the intermediate crystallinity (30-40\%) percolation takes place, and the cross-linking network becomes harder, manifesting the switch to the thermotropic behavior of the material. Simultaneously the divide between cross- and sliplinks becomes substantial, as an extensibility grows sharply.
\end{abstract}




\section{Introduction}

Nanoscale microphase separated systems attract a great attention in both academic and applied research circles in last decades[1,2,3]. Recent achievements in the chemistry of polyolefines stipulated by the progress in the design of new catalytic systems, allow synthesis of polymers with highly defined molecular structure, molecular weight distribution, and correspondingly with well-defined supramolecular organization, degree of crystallinity and a wide range of adjustable properties - from rubber-like elastomers to highly crystalline thermoplastics. Low crystalline materials with the crystallinity less than $10 \%$ exhibit pronounced ability to large reversible deformations while highly crystalline ones are able for only limited deformations because in such systems deformation behavior is determined by the changes in crystalline phase. Thus a unique opportunity to adjust material properties lies in variation of polymer crystallinity: with its increase formation of continuous crystalline phase (percolation) leads to the switch from typical rubber-like behaviour characterized by large, uniform, reversible deformations, to the typical drawing when necking occurs.

Changes in the molecular structure of polymers lead to the multiphase morphology of material, its complex crystallization behaviour and to significantly different properties from those of conventional polyolefines. Numerous efforts were devoted to study large deformations of such microheterogeneous polymer systems $[4,5,6,7,8]$, and to the establishment of relationships between the chemical structure of polyolefines and their physical characteristics determining possible commercial applications. However, such relationships are still not completely understood $[9,10,11]$.

Various models could be used to describe the high deformation of semicrystalline polymers. Recent developments in the interpretation of mechanical behaviour of thermoplastics have increasingly utilized a concept of the polymer chain conformation[12,13,14,15,16]. In its application to solid materials it is common to employ the hypothesis of Haward and Thackray[16] that elastic and viscous forces may be modelled in parallel. This is especially true for semicrystalline polymers where a consistent account was provided[17,18] for large strain properties in terms of entropic (chain straightening) and viscous (interchain friction) properties using nonGaussian chain statistics derived from inverse Langevin function[19]. Their treatment makes it possible to represent not only deformations measured in tension, but also those resulting from the application of other types of stress such as compression and shear.

From experimental point of view, powerful combination of deformation calorimetry and wide and small angle X-ray analysis allows to establish direct relationships between the molecular response on deformation and changes at the macroscopic level[20]. Based on these data it is possible to elucidate important aspects of the mechanical behaviour of polymer systems such as energy contribution to the elasticity of a rubber matrix, limited chain extensibility, reversibility of deformation, hysteresis 
phenomena during cyclic deformation, and the role of hard domains in the process of high elongation. It is also possible to distinguish the phase transitions and to study stress-induced crystallization and to investigate the nature of cross-link networks in the polymers.

Over the last three decades the tailored synthesis of PP in terms of defined stereoregularity evolved remarkably, facilitating a wide range of materials spreading from highly ordered plastics to low crystalline elastomers. Especially the latter aroused huge interest, since this polymer class is not accessible using classic Ziegler-Natta type systems[21,22,23] Cl symmetric complexes based on an ethylene-bridged fluorenyl-indenyl ligand framework (metallocene catalyst M) already demonstrated their high potential in polymer catalysis from elastic to thermoplastic polypropylene[6,24,25,26]. Approaches to isotactic polypropylene are generally conducted using heterogenic Ziegler-Natta type catalysts, although metallocene systems are nowadays available exceeding the heterogeneous ones in terms of stereo- and regioregularity as well as the potential to catalyze ultra-high molecular weights[27,28].

Isotactic polypropylene (iPP) is characterized by a rich variety of polymorphic transitions. Its macromolecular chains form $3_{1}$ helices with a period of $6.5 \AA$. They can be packed into three different crystalline phases: monoclinic $(\alpha)$, hexagonal $(\beta)$, and orthorhombic $(Y)$. Furthermore, the disturbance of the packing in each of those phases could be followed by the appearance of a mesophase[29]. Isotactic PP obtained with the use of conventional heterogeneous Ziegler-Natta catalysts usually forms the most common $\alpha$ phase $\left(a=6.65 \AA, b=20.96 \AA, c=6.50 \AA, \beta=99.3^{\circ}[30]\right)$. Its main feature is bilayer chain packing, in which individual layers are composed of a series of alternating left and right helices. The direction of the bilayers coincides with a crystallographic $b$ axis. Formation of $\mathrm{y}$ phase $(\mathrm{a}=8.54 \AA$, $\left.b=9.93 \AA, c=42.41 \AA^{\circ}[31,32,33]\right)$ is typical for the crystallization of iPP at high pressures. Moreover, it could be found more easily in a very low molecular weight iPP, in its copolymers, and/or when significant number of chain stereo- and regiodefects is present. In $\mathrm{Y}$ phase, neighbouring chains in the adjacent sublayers are not parallel to each other, as they are in $\alpha$ phase, but tilted at an angle of $\sim 81^{\circ}$, the angle complimentary to the monoclinic angle of the $\alpha$ phase. Thermal prehistory of the material plays crucial role in the crystallization mode of the material. For instance, nonisothermal crystallization of iPP leads to the formation of the $\gamma$ phase at high cooling rates or, in the thin films of low molecular weight material, to the formation of mesophase. For low cooling rates, the higher the concentration of chain structural defects, the higher content of crystallites of the $Y$ form $[34,35,36,37]$.

Systematic analysis of the structure of polypropylene samples with different stereoregularity, degree of orientation and thermal prehistory was 
carried out by us earlier[38]. It was revealed that the crystallites of the $\gamma$ modification form as the result of annealing of oriented samples at temperatures close to the melting one. Their contents are smaller at higher degrees of crystallinity of the as-prepared stretched films (e.g., at higher levels of stereoregularity of the material).

As (00l) planes of the $\gamma$ phase and $(0 \mathrm{k} 0)$ planes of the $\alpha$-phase are oriented in the same way, epitaxial crystallization of $\gamma$ modification crystallites takes place on the sidewalls of $\boldsymbol{\alpha}$-phase lamellas. Their formation is related to the existence of a small amount of unmelted oriented crystal fibrils in the partial melt. Orientation implies the existence of continuous lattice, with the crystallites playing a role of its joints. During melt crystallization, $\alpha$-phase lamellas appear. Their long axes are perpendicular to the direction of orientation, and iPP macromolecules are perpendicular to the plate surfaces. These deep structural transformations are possible only if there is a large quantity of the partial melt at the maximum annealing temperature.

One can expect that the crystallites of different types will play different roles in the mechanical behaviour of the material. In the present study we suppose to provide a detailed analysis of the morphology of PP samples with varied degree of isotacticity, and to establish relationships between the morphology of such materials and their mechanical properties. Obtained experimental data will be treated within the framework of conventional rubber elasticity theory, along with more advanced hypotheses (slip-link, tube model, etc.) in order to develop a theoretical description of the entire stress-strain curve and to elucidate the effect of different crystal junctions on the elastic properties of materials. 
Table 1: Structural and Thermal Characteristics of PP Samples.

\begin{tabular}{c|c|c|c|c|c|c|c|c|c}
\hline $\mathrm{N}$ & Sample & Isotacticity, $\%$ & $M_{w} \times 10^{5}$ & $\rho, \mathrm{g} / \mathrm{cm}^{3}$ & $\Delta H, \mathrm{~J} / \mathrm{g}$ & $X_{\gamma}, \%$ & $K_{p}, \%$ & $K_{\rho}, \%$ & $K_{\Delta H} \%$ \\
\hline 1 & M25-S & 25 & 1.5 & 0.855 & 5.0 & 0 & 0 & 6 & 3 \\
2 & M29-S & 29 & 10 & 0.854 & 0 & 0 & 0 & 5 & 0 \\
3 & M50-S & 50 & 0.8 & 0.880 & 54.5 & 89 & 38 & 38 & 33 \\
4 & M72-S & 72 & 10 & 0.897 & 89.1 & 82 & 62 & 59 & 54 \\
5 & M78-S & 78 & 13 & 0.900 & 87.5 & 90 & 59 & 63 & 53 \\
6 & M82-S & 82 & 2 & 0.900 & 90.8 & 79 & 63 & 63 & 55 \\
7 & M95-S & $>95$ & 15 & 0.910 & 113.0 & 29 & 69 & 73 & 69 \\
8 & ZN95-S & $>95$ & 3 & 0.915 & 145.2 & 19 & 75 & 77 & 88 \\
\hline 1 & M25-Q & 25 & 1.5 & 0.854 & 0 & 0 & 0 & 5 & 0 \\
2 & M29-Q & 29 & 10 & 0.856 & 0 & 0 & 6 & 7 & 0 \\
3 & M50-Q & 50 & 0.8 & 0.873 & 44.6 & 0 & 29 & 28 & 27 \\
5 & M78-Q & 78 & 13 & 0.883 & 54.0 & 0 & 35 & 41 & 33 \\
6 & M82-Q & 82 & 2 & 0.880 & 63.0 & 0 & 44 & 37 & 38 \\
7 & M95-Q & $>95$ & 15 & 0.896 & 84.2 & 0 & 52 & 56 & 51 \\
8 & ZN95-Q & $>95$ & 3 & 0.900 & 97.4 & 0 & 64 & 61 & 59 \\
\hline
\end{tabular}

Isotacticity is the content of [mmmm] pentads in the corresponding samples, $\rho$ is their macroscopic density, $\Delta H$ is the fusion heat obtained by the method of differential scanning calorimetry, $X_{\gamma}$ and $K_{p}$ are the content of $\gamma$-phase and sample crystallinity obtained from the analysis of WAXS-patterns, $K_{\rho}$ and $K_{\Delta H}$ are the crystallinities calculated from the 
macroscopic density and fusion heat of the samples correspondingly. Heat of fusion of $165 \mathrm{~J} / \mathrm{g}$ for crystalline iPP was used $\left.{ }^{39}\right]$. 


\section{Experimental}

PP samples with various degrees of stereoregularity were synthesized in the presence of metallocene catalysts (M)[40]. The content of [mmmm] pentads was equal to $25,29,50,78$ and more than $95 \%$, polydispersity of materials being $\mathrm{Mw} / \mathrm{Mn} \sim 2$. The detailed characterization of the studied samples, including their thermal and structural parameters, could be found in Table l. Additionally, PP with an isotacticity of more than 95\% was synthesized using Zigler-Natta catalysts, designated hereinafter as ZN-95.

Actual samples were produced as films $\sim 0.5 \div 0.7 \mathrm{~mm}$ thick via melt compression at a pressure of $15 \mathrm{MPa}$, followed either by quenching in the ice water [37,38] (additional postfix Q will be used in the designations of such samples), or by slow cooling with an average rate of $3^{\circ} \mathrm{C} / \mathrm{min}$ (corresponding postfix $\mathrm{S}$ in the designations). Depending on the degree of isotacticity, the compression temperature was varied in the range of $100 \div 250^{\circ} \mathrm{C}$.

Samples for mechanical tests were prepared as dumbbell shaped films, their working parts being $5 \mathrm{~mm}$ x $20 \mathrm{~mm}$. They were stretched at a rate of 10 $\mathrm{mm} / \mathrm{min}$ using Instron-6022 testing machine at room temperature until fracture. True degree of deformation $\lambda$ was determined via labeling. Amorphous samples M25 and M29 remained translucent after stretching. Deformation of M25 was accompanied by flow, while in M29 it was fully reversible, and prolonged exposure to the isometric conditions at room temperature resulted in the sample fracture. The results of measurements were averaged from the data from $5 \div 8$ samples.

In the process of sample deformation, series of photographs of the ink grid were taken by Nikon Coolpix 4500 camera. Special procedure of image treatment allowed us to determine real deformations and real cross-sections of deformed films in certain moments. These data were used to reconstruct the true stress-strain curves. It was revealed that local deformations can be very different throughout the sample volume not only at the stages of neck formation and of its propagation, but the regions with different draw ratios can always be easily distinguished. The true stress was calculated for the minimal cross-section. As a rule, this region corresponds to the site of necking. Two examples of the calculation of true stress-strain curves using the obtained engineering curves, are shown on Figure 1.

DSC measurements were performed using Mettler DSC-30 and Perkin-Elmer DSC-7 instruments. As-prepared films of $2 \div 10 \mathrm{mg}$ weight were heated from $-150^{\circ} \mathrm{C}$ to $+250^{\circ} \mathrm{C}$ with the rate of $20^{\circ} \mathrm{C} / \mathrm{min}$. Degree of crystallinity was calculated as a ratio of the heat of fusion of given sample to that of an ideal PP crystal $(\Delta \mathrm{Ho}=165 \mathrm{~J} / \mathrm{g})$. To elucidate an effect of preparation conditions on crystallinity and thermal behavior of original pellets, different types of samples were studied: 1) films quenched in a mixture of water and ice; 2) slowly crystallized films. 

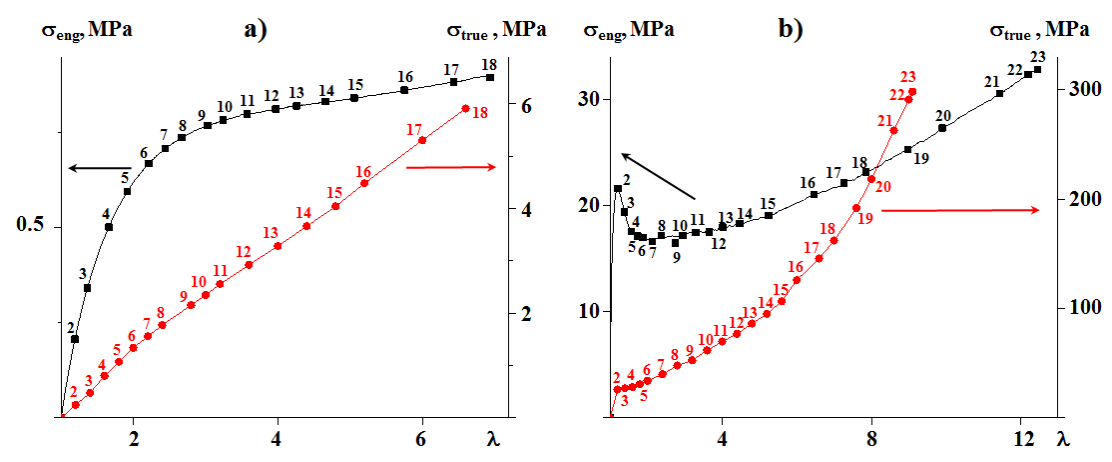

Figure 1: Comparison of engineering (black squares) and true (red circles) for M29-S and M72-S samples.

Detailed characterization of the structure of as-prepared PP films and of its transformation in the process of deformation was carried out by $\mathrm{X}$-ray scattering analysis. Linear equatorial and meridian diffraction scans of drawn fibers were recorded in the transmission mode with the use of DRON-3 instrument (CuK $\alpha$ radiation, curved quartz crystal monochromator). Degree of crystallinity of as-received samples was estimated from wide angle data as a ratio of the integral intensity of crystalline diffraction peaks to the total scattering, after the subtraction of instrumental background and correction for Lorentz and polarization factors. The lateral and longitudinal crystallite sizes were obtained from the integral half-widths of corresponding reflections using Sherrer equation after a correction for instrumental broadening. Structural changes on supramolecular hierarchical level in the process of sample deformation were monitored by small angle $\mathrm{X}$-ray scattering using compact Kratky camera (Ni-filtered $\mathrm{CuK} \alpha$ radiation). The measurement of absolute intensity of SAXS based on secondary standard was used to determine the density of crystalline and amorphous regions [41]. For the system containing two phases of different electron densities $\eta_{a}$ and $\eta_{b}$, mean square fluctuation of electron density is

where

$$
\left\langle\Delta \eta^{2}\right\rangle=\left\langle(\eta-\bar{\eta})^{2}\right\rangle=\left(\eta_{a}-\eta_{b}\right)^{2} \cdot w_{a} w_{b}
$$

$$
\bar{\eta}=\eta_{a} w_{a}+\eta_{b} w_{b}
$$

Equation (l) is directly related to the scattering invariant $\tilde{Q}$ of the slit collimated scattering curve:

$$
\tilde{Q}=\int_{0}^{\infty} \tilde{I}(s) \cdot s d s=4 \pi^{2} \cdot \widetilde{I_{0}} \cdot \frac{e^{4}}{m^{2} c^{4}} \cdot \frac{1+(\cos 2 \theta)^{2}}{2 a^{2}} \cdot V \cdot\left\langle\Delta \eta^{2}\right\rangle
$$

Here $\widetilde{I}_{0}$ is an intensity of the primary beam, $V$ is the irradiated volume, a is sample - detector distance, $s$ is a wave vector modulus:

$$
s=\frac{4 \pi \cdot \sin \theta}{\lambda}
$$

and

is the scattering power.

$$
\frac{e^{4}}{m^{2} c^{4}} \cdot \frac{1+(\cos 2 \theta)^{2}}{2 a^{2}} \cdot V \cdot\left\langle\Delta \eta^{2}\right\rangle=\tilde{P}
$$


Intensity of the primary beam was measured using secondary polyethylene etalon[42] with intensity maximum at $0.02115 \AA^{-1}$. It is known that in oriented samples mean square fluctuation of the electron density fluctuation along fibrillae is given by[43] the relationship:

$$
\left\langle\Delta \eta^{2}\right\rangle=\frac{1}{2 \pi} \frac{m^{2} c^{4}}{e^{4}} \cdot \frac{a}{I_{0} V \lambda N_{a}^{2}} \cdot \int \widetilde{I}_{\|}(s) \cdot s d s
$$

where $\lambda$ is an X-Ray wavelength and $N_{a}$ is Avogadro number.

Electron density $\eta$ and mass density $\rho$ correlate to each other:

$$
\eta=\frac{\sum_{i} Z_{i}}{\sum_{i} M_{i}} \cdot \rho
$$

Here $Z_{i}$ and $M_{i}$ are the charge and mass of the $t^{\text {th }}$ atom of the repeating unit. One can obtain for a two phase system

$$
\left\{\begin{array}{c}
\rho=\rho_{a} w_{a}+\rho_{b} w_{b} \\
\left\langle\Delta \eta^{2}\right\rangle=(\Delta \eta)^{2} \cdot w_{a} w_{b}
\end{array}\right.
$$

The density of the amorphous phase was calculated by taking into account the macroscopic density and the sample degree of crystallinity. Long period of microfibrillar structure was calculated with the use of Bragg equation after application of the desmearing procedure by SYRENA program.

\section{Structure of the materials}

It is interesting to note that the structure of samples obtained by slow cooling and quenching of the melt, differs substantially - in addition to significant differences in the supramolecular structure (size and / or defects in the crystallites), they differ in phase composition. The former contains a mixture of $\alpha$-and $\gamma$-crystalline modifications phases, while in the latter one only mesophase and the traces of $\alpha$-phase were observed.

WAXS-patterns of slowly cooled samples ZN95-S and M95-S reveal a number of rather narrow reflections. The higher the quantity of stereodefects in the materials, the wider the observed reflections, as the crystallites become smaller in size and less perfect in the ordering. The only exception is the diffraction maximum at $\mathrm{q}=1.2 \AA-1$ whose half-width does not vary from sample to sample. Moreover, the content X $\gamma$ of $\gamma$-phase grows as well - from $19 \%$ in the ZN95-S to $90 \%$ in M78-S and M90-S (see Table 1). The majority of the reflections of $\gamma$-modification overlap with those of $\alpha$-phase leading to the seeming redistribution of the intensities of the observed reflections. The only separate reflections allowing the reliable estimation of phase content are $130^{\alpha}$ and $117^{\gamma}$. We should also pay our attention on the effect of molecular weight on the crystallization of isotactic polypropylene. The content of $\gamma$-phase is abnormally high in the high molecular weight M78-S samples as the longer molecules are more prone to the formation of different kinds of topological defects which in turn help to deviate molecular chain helices from their parallel packing in the crystalline packing of the $\alpha$-phase. WAXS-patterns of M29-S and M25-S samples show only the presence of a wide asymmetric amorphous halo. 
WAXS-patterns of quenched samples reveal substantially wider overlapping crystallographic reflections, as the formed crystallites are much less perfect (Figure 2b). As the most intense reflection of the $\gamma$-phase $117^{\gamma}$ is absent, we can make a conclusion that the preferred mode of the crystallization in the quenched samples is through the formation of $\alpha$ modification and of mesophase.

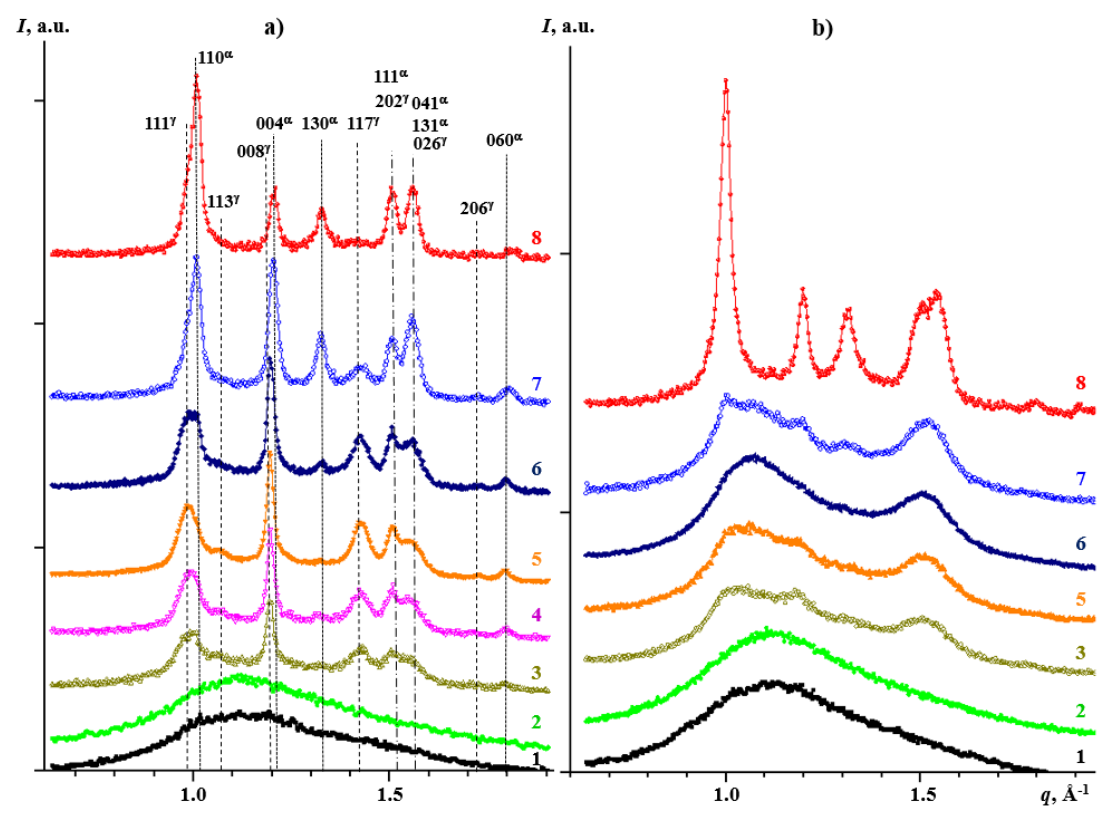

Figure 2: WAXS-patterns of slowly cooled (a) and quenched (b) samples of polypropylene with different degrees of isotacticity: M25 (1), M29 (2), M50 (3), M72 (4), M78 (5), M82 (6), M95 (7) and ZN95 (8). Indexing of the reflections of a- and gamma-phases is shown as well.

Average effective sizes of crystallites were estimated using Sherrer equation from the integral half-width of the second narrow intensive maximum containing 040 reflection of the $\alpha$-phase and 008 one of the $\gamma$ modification. They were found to be around $-30 \mathrm{~nm}$. Fixed position of this line and comparatively low value of its small half-width suggest that the interplanar distances $040^{\alpha}$ and $008^{\gamma}$ are virtually independent of the stereoregularity of polymer chains. It seems tempting to estimate the lateral crystallite sizes in the studied samples from the first diffraction maximum, containing two overlapping diffraction lines $110^{\alpha}$ and $111^{\gamma}$. However, different (and varying from sample to sample) positions as well as intensities of those two reflections do not allow their reliable deconvolution. For most regular samples M95-S and ZN95-S, lateral dimensions of the $\alpha$-phase crystallites can be estimated from the half-width of separate reflection $130^{\alpha}$ (24 and $28 \mathrm{~nm}$ respectively). Samples of lower crystallinity such as M50-S and M82-S, are characterized by the high content of the $\gamma$-modification. So it is possible to estimate the lateral sizes of its crystallites from the half-width 
of $117^{\gamma}$ reflections, they were found to be equal to $10-14 \mathrm{~nm}$. Thus, in the iPP samples of different stereoregularities one can find $\alpha$ - and $\gamma$-phase crystallites which are highly ordered only along the direction perpendicular to the planes of bilayers: $0 k 0^{\alpha}$ and $00 l^{\gamma}$ respectively. This fact can be explained by the specifics of polypropylene crystallization. The basic structural unit of all polypropylene crystalline modifications is a bilayer composed of left-handed and right-handed helices. Macromolecular chains in neighboring bilayers of the $\alpha$-phase are parallel to each other and characterized by a regular alternation of right-hand and left-hand helices, while in the $\gamma$ phase neighboring chains in adjacent bilayers have similar rotation directions of helices. As the degree of isotacticity decreases and both translational and rotational defects in chain packing within bilayers arise, the order in the perpendicular direction remains virtually unchanged. In the quenched PP samples, the development of the mesophase is also related to the absence of any correlation between neighboring layers.

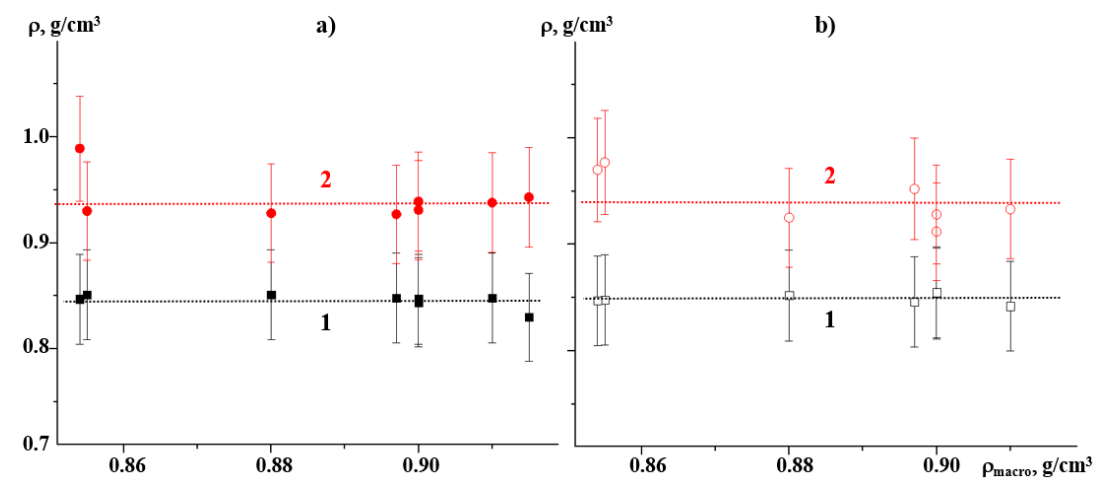

Figure 3: Densities of the amorphous (1) and crystalline (2) phase in the slowly cooled (a) and quenched (b) samples.

Certainly, different kinds of crystallites - mesophase, $\alpha$ - and $\gamma$-phase, must serve as different types of the links in the cross-linking net. However, we calculated the densities of amorphous and crystalline phases using the absolute measurements of the intensity of small-angle X-Ray scattering. As Figure 3 reveals, the values of both amorphous and crystalline does not change with material crystallinity. As quenched samples of intermediate crystallinity contain the majority of mesophase, one can make a conclusion that its density is close to that of crystalline $\alpha$-modification.

\section{Models of deformation}

Classical theory of the rubber-like elasticity is based on the assumption of Gauss distribution of the distance $r$ between the chain ends. In such case $r$ is substantially smaller than contour length of polymer macromolecule $r_{\max }$ [44]. The model explains relatively small deformations for polymer nets which are far from their utmost extended state. Polymer nets can be easily described by a three-chain model in which a real net is 
approximated by three independent sets of chains, every one of them is parallel to one of the axis of an orthogonal coordinate system. In nondeformed state radius-vectors of chain ends are oriented along $\mathrm{Ox}$, Oy or $\mathrm{Oz}$ axis and have the same length of $r_{0}$. If deformation is affine, their coordinates after a uniaxial orientation along $O x$ with a draw ratio of $\lambda$ are:

$$
r_{x}=\lambda r_{0} ; r_{y}=r_{z}=\frac{r_{0}}{\sqrt{\lambda}}
$$

For $n$ links with an average length of l, calculations of Kuhn and Grün give an overall entropy $S$ of such system as

$$
\begin{aligned}
& S=\left(s_{x}+2 s_{y}\right)=-k n\left\{\frac{r_{0} \lambda}{n l} L^{-1}\left(\frac{r_{0} \lambda}{n l}\right)+\ln \frac{L^{-1}\left(r_{0} \lambda / n l\right)}{\sinh L^{-1}\left(r_{0} \lambda / n l\right)}\right\}- \\
& -2 k n\left\{\frac{r_{0} \lambda^{-1 / 2}}{n l} L^{-1}\left(\frac{r_{0} \lambda^{-1 / 2}}{n l}\right)+\ln \frac{L^{-1}\left(r_{0} \lambda^{-1 / 2} / n l\right)}{\sinh L^{-1}\left(r_{0} \lambda^{-1 / 2} / n l\right)}\right\}
\end{aligned}
$$

If $\mathrm{N}$ is a number of chains in the unitary volume and $S_{0}$ is an entropy of a free state, then the deformation work is

$$
W=-T\left(S-S_{0}\right)=-T\left\{N \cdot \frac{\left(s_{x}+2 s_{y}\right)}{3}-S_{0}\right\}
$$

Deformation stress per unitary area is thus

$$
\sigma=\frac{\partial W}{\partial \lambda}=-\frac{N T}{3} \frac{\partial\left(s_{x}+2 s_{y}\right)}{\partial \lambda}=\frac{N k T}{3} \frac{r_{0}}{l}\left\{L^{-1}\left(\frac{r_{0} \lambda}{n l}\right)-\lambda^{-3 / 2} L^{-1}\left(\frac{r_{0} \lambda^{-1 / 2}}{n l}\right)\right\}
$$

Using Pade equation for the analytical approximation of Langevin function[45] and changing $r_{0}$ by an average square length $n^{1 / 2} l$ between the joints of the polymer net it is easy to obtain

$$
\sigma=(N k T / 3)\left[\left(\lambda\left(3-\lambda^{2} / n\right) /\left(1-\lambda^{2} / n\right)-\left(1 / \lambda^{2}\right)(3-1 / \lambda n) /(1-1 / \lambda n)\right)\right]
$$

It should be noted that one can't describe the whole deformation curve using only two constants $\mathrm{N}$ and $\mathrm{n}$, therefore additional constant $\mathrm{Y}_{0}$ treated as yield stress should be introduced. For rubbery materials the value of $\mathrm{Y}_{0}$ is generally small compared to the stress at high deformations. However, for thermoplastics $Y_{0}$ increases together with crystallinity and reaches its maximum at the moment of neck formation. Finally equation (12) is transformed to

$$
\sigma=Y_{0} / \lambda+(N k T / 3)\left[\left(\lambda\left(3-\lambda^{2} / n\right) /\left(1-\lambda^{2} / n\right)-\left(1 / \lambda^{2}\right)(3-1 / \lambda n) /(1-1 / \lambda n)\right)\right]
$$

This model developed by Haward describes successfully uniaxial deformation of a number of copolymers at high draw ratios [46]. All variables used for the fitting of true stress-strain curves have clear physical meaning and correlate with polymer crystallinity, molecular weight and deformation 
temperature. It should be emphasized that equation (13) describes the mechanical response of a homogeneous network with fixed links. It will be clear that such a description is only an approximation of the real mechanical response of linear short chain branched polyolefines, that might involve slipping entanglements, slipping chains and last but not least the network node activity of the crystals.

Another concept is based on the theory of elasticity using Gaussian approximation and accounting for the topological constraints in entangled networks, it was proposed by Doi and Edwards $[47,48]$. In this model, the free energy of single chains between the crosslinks is described by the common statistical theory, whereas entropy reduction due to entanglements with neighboring chains is described by a number of slip-links which are free to slide along the length of an active chain between cross-links. These slip links are basically moveable network junctions that are able to slip between fixed links and hence contribute to the entropic stress built up at strain values within the strain limits of Gaussian deformation. Edwards and Vilgis [49] extended the model of chain inextensibility and obtained:

$$
\begin{array}{r}
\frac{F}{k T}=\frac{1}{2} N_{s}\left[\sum_{i=1}^{3}\left\{\frac{\lambda_{i}^{2}\left(1-\alpha^{2}\right)(1+\eta)}{\left(1-\alpha^{2} \cdot \sum_{i=1}^{3} \lambda_{i}^{2}\right)\left(1+\eta \lambda_{i}^{2}\right)}\right\}+\ln \left(1-\alpha^{2} \cdot \sum_{i=1}^{3} \lambda_{i}^{2}\right)\right] \\
+\frac{1}{2} N_{c}\left[\frac{\sum_{i=1}^{3} \lambda_{i}^{2}\left(1-\alpha^{2}\right)}{1-\alpha^{2} \cdot \sum_{i=1}^{3} \lambda_{i}^{2}}+\ln \left(1-\alpha^{2} \cdot \sum_{i=1}^{3} \lambda_{i}^{2}\right)\right]
\end{array}
$$

where summing is performed over the three Cartesian components of strain, F is the free energy of the system, $N_{c}$ and $N_{s}$ are the densities of cross- and slip-links correspondingly, $\lambda$ is the draw ratio of the sample, $\eta$ is slippage parameter, and $\alpha$ is inextensibility parameter. If $\eta=0$, slip-links are rigid and act as cross-links. On the other hand, if $\alpha=0$, the equation is reduced to the standard Gauss statistics of ideal polymer chains. Differentiation of the last equation over the draw ratio gives components of the elastic force provided by the presence of slip-and cross-links respectively:

$$
\begin{aligned}
& f_{s, x}=\left(\frac{\partial F_{s}}{\partial \lambda_{x}}\right)_{T, V, \lambda_{y}, \lambda_{z}} \\
& =N_{s} k T \\
& \left(\begin{array}{c}
\frac{\eta\left(\lambda^{3}-1\right)}{\lambda\left(1+\eta \lambda^{2}\right)(\lambda+\eta)}-\frac{\alpha^{2}}{\left(1-\alpha^{2}\left(\lambda^{2}+\frac{2}{\lambda}\right)\right)} \cdot\left(\lambda-\frac{1}{\lambda^{2}}\right)\left[1-\frac{\left(1-\alpha^{2}\right)(1+\eta)}{\left(1-\alpha^{2}\left(\lambda^{2}+\frac{2}{\lambda}\right)\right)} \cdot \frac{\lambda^{3}+3 \eta \lambda^{2}+2}{\left(1+\eta \lambda^{2}\right)(\lambda+\eta)}\right] \\
+\frac{\left(1-\alpha^{2}\right)(1+\eta)}{\left(1-\alpha^{2}\left(\lambda^{2}+\frac{2}{\lambda}\right)\right)} \cdot \frac{-\eta^{2} \lambda^{4}+\lambda^{3}+\lambda \eta^{2}-1}{\left(1+\eta \lambda^{2}\right)^{2}(\lambda+\eta)^{2}}
\end{array}\right)
\end{aligned}
$$

$$
f_{c, y}=\left(\frac{\partial F_{c}}{\partial \lambda_{x}}\right)_{T, V, \lambda_{y}, \lambda_{z}}=N_{c} k T \cdot \frac{\left(\lambda-\frac{1}{\lambda^{2}}\right)}{\left(1-\alpha^{2}\left(\lambda^{2}+\frac{2}{\lambda}\right)\right)} \cdot\left\{\frac{\left(1-\alpha^{2}\right)}{\left(1-\alpha^{2}\left(\lambda^{2}+\frac{2}{\lambda}\right)\right)}-\alpha^{2}\right\}
$$


Considering sample volume constant and deformation uniform, one can obtain true stress components $\sigma_{\mathrm{s}}$ and $\sigma_{\mathrm{c}}$ by using Equations (8) and thus simply multiplying Equations (15) and (16) by the draw ratio.

It's worth noting that each of the described approaches has its own advantages and failures. Non-Gaussian concept by Haward offers a rather simple semi-empirical treatment allowing a comprehensive coverage of the whole deformation process using, however, a rather voluntary parameter of yield stress is introduced. In contrast, slip-link model extrapolates the whole stress-strain dependence, though its equation is comparatively complex and introduces larger number of parameters which a more difficult to derive from an experiment. Fitting of the experimental true stress-strain curves is though rather straightforward procedure, as different parameters make substantially different effects on the experimental curves, so it is quite easy to fit them using simple least square root procedure.

Obviously, the employment of rubber elasticity theory outside the specific circumstances for which it was developed, raises serious questions. For example, the classical theory of rubber elasticity is predicated by the existence of long polymer chains where bond rotation occurs freely without significant energy barriers or frictional interaction between the moving chains. A further requirement is that the polymer chains are linked by covalent bonds unaffected by changes in temperature in the range of interest. Calculations based on the configurational entropy of the chains then generate equations, which describe the well-known characteristics of reversible rubber elasticity including the proportionality of stress to absolute temperature. In our work both theoretical methods were employed to clarify the mechanism of large deformations in polypropylene samples of different molecular structure.

\section{Analysis of the mechanical behavior}

True stress-strain curves obtained as was described in the Experimental part, are presented on Figure 4. One can see that deformational behavior of the samples depends strongly of the degree of stereoregularity and of the thermal prehistory of material as well. Deformation of highly crystalline samples M95-S and ZN95-S did not exceed 25\%. The most pronounced necking was observed on the deformation of ZN95-Q and M95$\mathrm{Q}$ samples; it was accompanied by an intense pore formation. The neck grew at low deformations corresponding to the yield stress on experimental stress-strain curves.

Deformation of M82 - M50 samples was not uniform as well both for quenched and slowly cooled samples. After the growth of pronounced neck, oriented parts of samples were elongated, with the maximal draw ratio of 9 . Comparing, however, the samples of the same degree of isotctacticity and of different thermal prehistory, one can make an observation that deformation of slowly cooled films is less uniform. This fact can be explained by a higher 
degree of crystallinity in the slowly cooled samples, and, correspondingly, by a higher number and/or size of crystallites (see Table 1). After the necking, the samples became much more opaque due to the destruction of lamellar structure and/or of cavitation taking place within amorphous phase $\left[{ }^{50}\right]$.
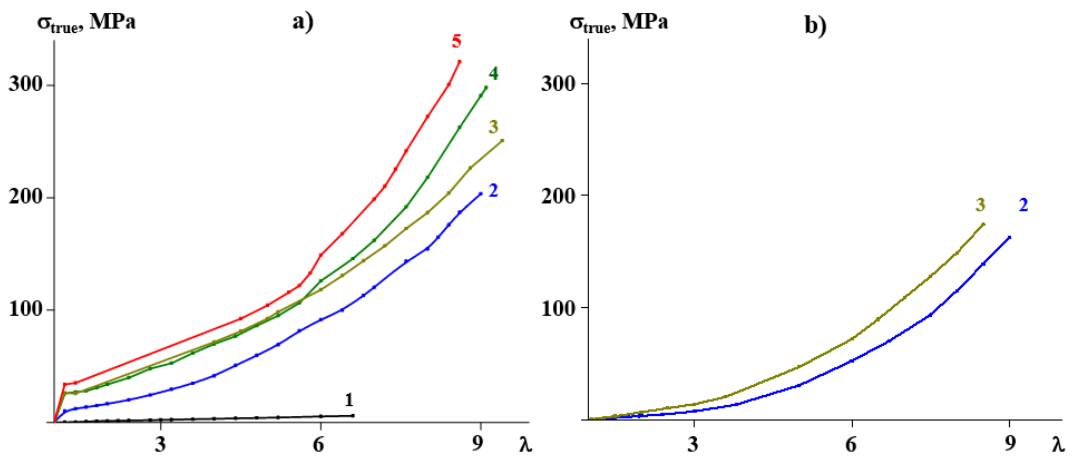

Figure 4: True stress-strain curves for slowly cooled (a) and quenched (b) samples of M29 (1), M50 (2), M72 (3), M78 (4) and M82 (5) samples.

Almost fully amorphous films M25 and M29 could be extended in the most uniform way. As the isotacticity changes from $29 \%$ to $50 \%$, the neck appears (and the yield stress swings upwards) manifesting the change of the mechanical behaviour of the material from elastomeric to a thermoplastic one. Such effects are more pronounced in the slowly cooled films, as the crystallinity of the quenched samples is much lower. An effect of molecular weight on the mechanical behaviour of iPP is most apparent on the comparison of M25-Q and M29-Q samples. Deformation fracture of low molecular weight $(\mathrm{Mw}=150000) \mathrm{M} 25$ samples calculated for the regions between $1 \mathrm{~mm}$ marks on the as-received samples (see Experimental), reached $\lambda \sim 100$, whereas neighboring regions had an elongation of just 3-5. After the fracture, the residual strain in the neck remained rather high exceeding $\lambda-50$. One could explain this effect by the flow of the material in the neck, while other parts of the sample does not gain any deformation or even relax. The higher molecular weight M29-Q samples had the same crystallinity (about $5 \%$ according to their macroscopic density), though their deformation was substantially more uniform and reached the draw ratio value of 7-8.

The relation between the structure of the polymer and mechanical properties of the samples can be better understood if, instead of the degree of isotacticity one could use crystallinity of the material. The latter characteristics, on the one hand, is directly dependent of the degree of isotacticity. On the other hand, it is one of the most factors determining the exploitation properties of polymer products. Figure 5 shows the dependence of iPP Young modulus of its crystallinity calculated from the macroscopic density of the samples. The data can be approximated by the dashed curves that are very characteristic for percolation mechanisms. The bend of the curve is in the region around $40 \%$ of crystallinity. 


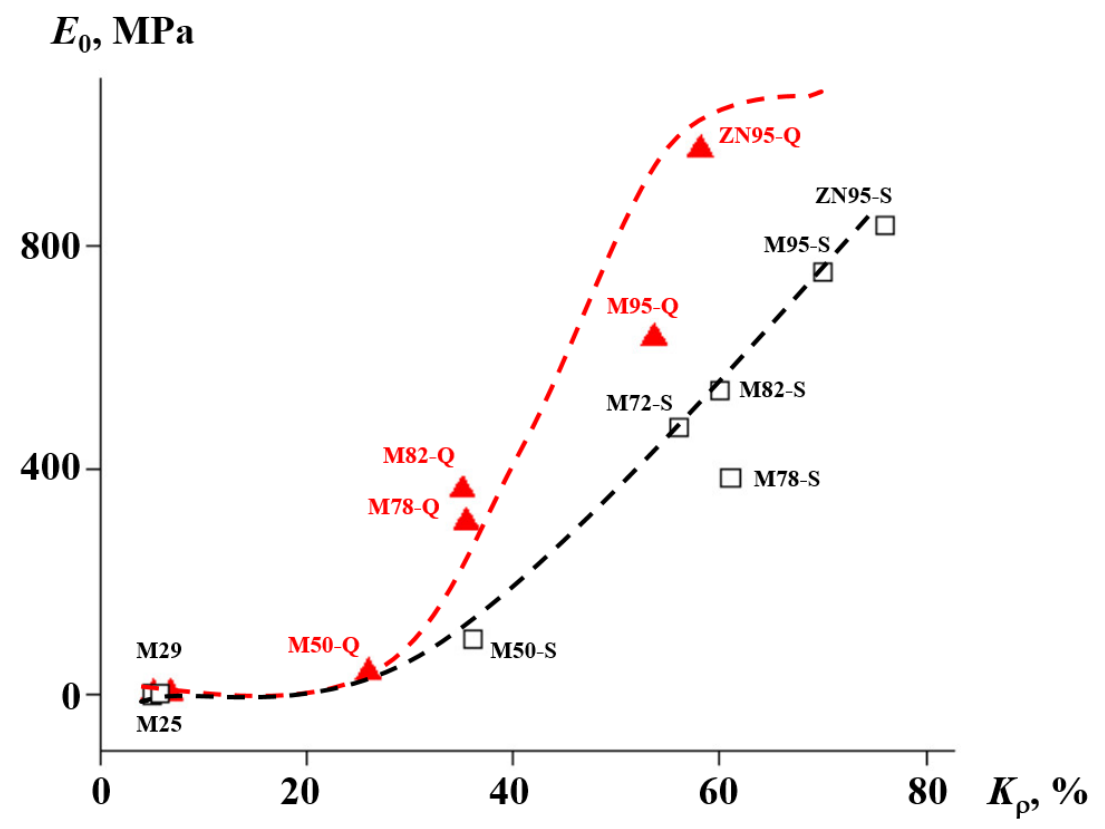

Figure 5: Dependence of iPP Young modulus of its crystallinity calculated from the macroscopic density of the samples.

In our case one can suppose that in the region of the bend of $E_{0}\left(K_{\rho}\right)$ dependencies, percolation takes place when the density of cross-links becomes such that the links no longer feel each other at further separation. Thus, mechanism of deformation and, correspondingly, mechanical behavior of the material should change in the aforementioned range of crystallinities. Moreover, an obvious conclusion follows, that, as the percolation manifests itself on the crystallinity dependencies, the role of the cross-links is played by the crystallites theirselves. To prove our statements, we should establish correlations between the structure and characteristics of the cross-linking nets in the studied samples.

On the first stage we applied Haward approach with the aim of quantitative description of the physical cross-linking nets in the polypropylene with different degrees of isotacticity. It allows deriving the number $\mathrm{N}$ of the effective cross-links (hereafter NkT which is equivalent to the shear modulus $G$ ) and the concentration $n$ of elastic chains between those cross-links. Figure 6 shows several examples of the true stress-strain curves in Gaussian coordinates $\sigma_{t r}\left(\lambda^{2}-1 / \lambda\right)$. One can see that the samples from the lower base of $\mathrm{S}$-shaped $E_{0}\left(K_{\rho}\right)$ dependence (Figure 6a) show no deviation of the straight line of Gauss law, allowing the determination of the modulus $\mathrm{G}$ which is equal to $0.08 \mathrm{MPa}$ for M25-S and 0.1 MPa for M29-S samples. However, the higher the crystallinity of the material, the stronger the upswing deviation of the true curves from Gaussian law, as the neighboring cross-links make the growing contribution to the limited elasticity. Moreover, in the quenched samples (Figure 6d) deviations from 
the Gaussian plots are substantially higher than those in the slowly cooled ones. This fact is easily explainable, as quenching causes the increase of the density of cross-linking net. The parameters of cross-link nets in the studied samples, calculated from stress-strain curves, are presented in Table 2.

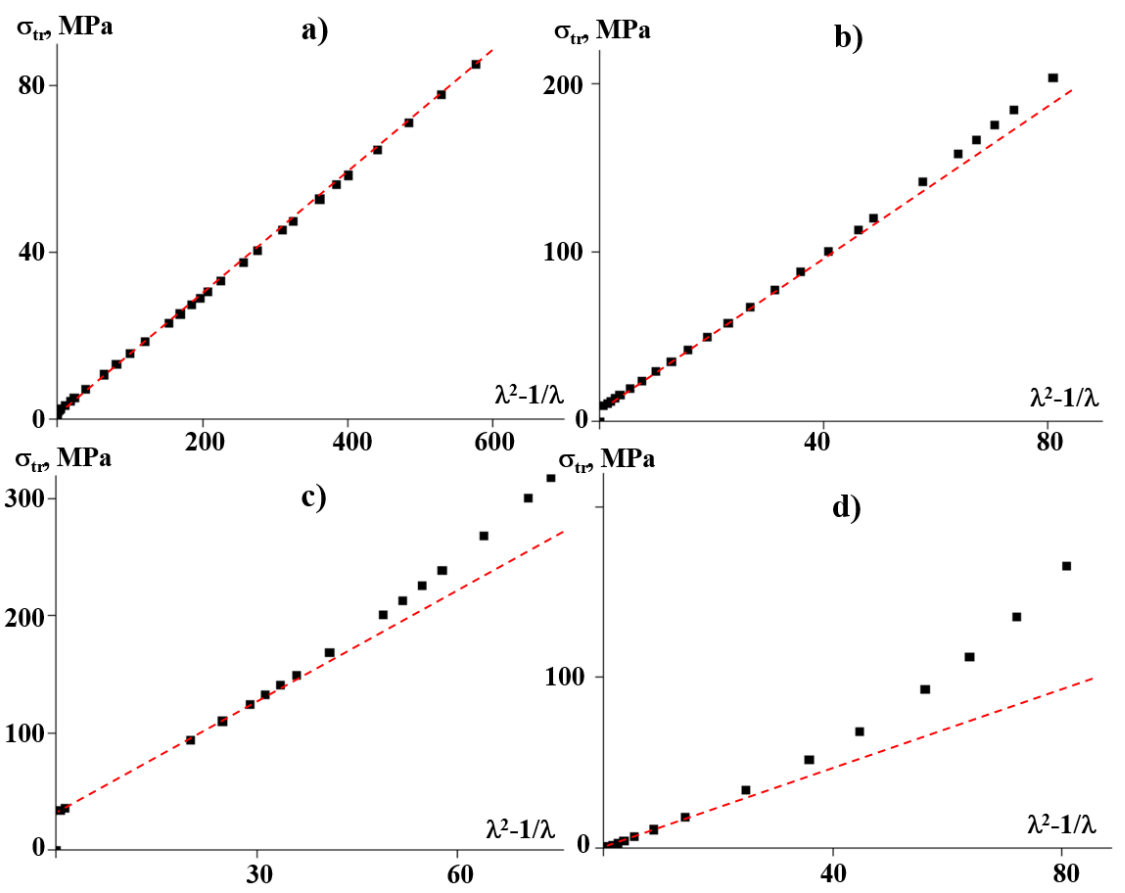

Figure 6: Gaussian plots for M25-S (a), M50-S (b), M82-S (c), M50-Q (d) samples of iPP.

As one can see, the values of $\mathrm{NkT}$ in the slowly cooled samples grows together with crystallinity from 0.08 MPa (M25-S) to 2.9 MPa (M82$S$ ). The values of $\mathrm{NkT}$ in the quenched samples are substantially lower than those in the slowly cooled materials with the same degree of isotacticity. This fact once more proves the conclusion that it is the crystallinity of the material which effects directly their mechanical behavior. The average length $n$ of free chains has a trend of the decrease with the growing isotacticity from 6250 in M25-S to 220 in M82-S. However, it is also strongly affected by the molecular mass of the polymer (see comparatively low values of $n$ for high molecular weight materials M29 and M72). We can as well underline a high efficiency of the process of quenching as a decreasing factor for $n$ (160 and 192 in M50-Q and M72-Q correspondingly) due to an increased possibility of formation of topological constraints.

Table 2: Parameters of the cross-link nets obtained using Haward model.

\begin{tabular}{|c|c|c|c|}
\hline iPP & Y, MPa & G, MPa & n \\
\hline M25-S & 2 & 0.08 & 6250 \\
\hline M29-S & 2 & 0.10 & 220 \\
\hline
\end{tabular}




\begin{tabular}{|c|c|c|c|}
\hline M50-S & 7.9 & 2.15 & 515 \\
\hline M72-S & 27.5 & 2.30 & 210 \\
\hline M78-S & 30 & 2.3 & 640 \\
\hline M82-S & 32 & 2.90 & 220 \\
\hline & & & \\
\hline M50-Q & 0 & 1.215 & 160 \\
\hline M78-Q & 1.35 & 1.73 & 192 \\
\hline
\end{tabular}

Application of the model of the slip-links must clarify the role of the structural elements in the mechanical behavior of the materials. According to this approach, crystallites may play a role of the soft, labile slip-links, while the role of the cross-links with the limited extensibility $\alpha$ is served by the topological ties of macromolecular chains or folded chain crystallites. As it was described above, there are four variable parameters in the model of sliplinks. Together with $\alpha$, they are slippage parameter $\eta$ and concentrations of cross- and slip-links $N_{c}$ and $N_{s}$ respectively. In our approximations of the true stress-strain curves we varied three of them (see Table 3, Figure 7) supposing $\eta$ to be 1.1, as it was found for the linked polyethylene fibers extended from the melt [51].

Table 3: Parameters of the cross-link nets obtained using slip-link model.

\begin{tabular}{|c|c|c|c|}
\hline iPP & NskT, MPa & NckT, MPa & $\boldsymbol{\alpha}$ \\
\hline M25-S & 7.0 & 0.11 & 0.012 \\
\hline M29-S & 3.6 & 0.09 & 0.009 \\
\hline M50-S & 30.4 & 0.69 & 0.038 \\
\hline M72-S & 87.9 & 1.08 & 0.058 \\
\hline M78-S & 97.4 & 1.20 & 0.044 \\
\hline M82-S & 95.2 & 1.36 & 0.061 \\
\hline & & & \\
\hline M50-Q & 3.0 & 1.14 & 0.056 \\
\hline M78-Q & 4.0 & 1.66 & 0.048 \\
\hline
\end{tabular}



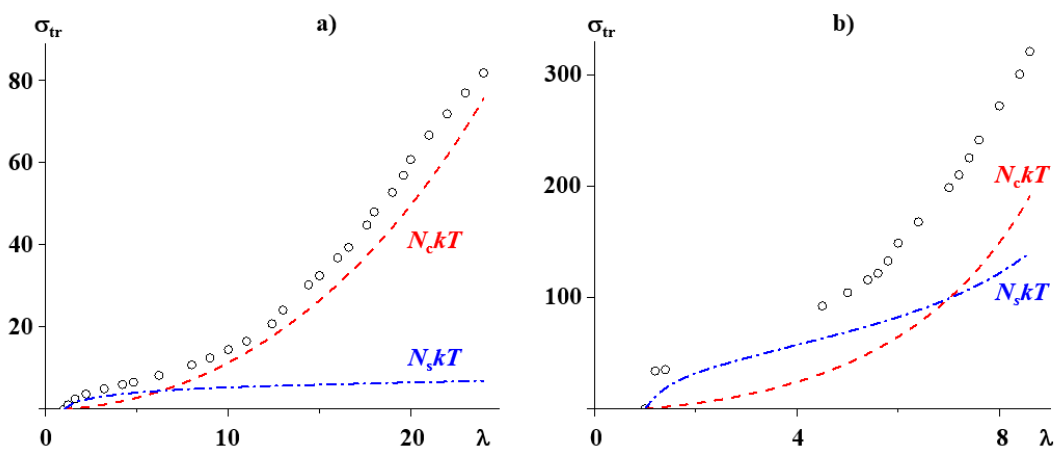

Figure 7: Contributions of cross-links and slip-links into true stress-strain curves for M25-S and M82-S samples.

As Table 3 reveals, both the values of $N_{s} \mathrm{kT}$ and $N_{c} \mathrm{kT}$ depend on the crystallinity and grow with the increased degree of isotacticity. Between M29-S and M82-S, the former has grown by 26 times, and the latter one - by 15 times. Moreover, parameter of the limited extensibility also increases sharply near the percolation limit. These results suppose that really crystallites play not only the role of slip-links, but also often act as crosslinks as well. Hence the growth of the parameter $\alpha$, as the crystallites start feeling each other near the percolation limit, and thus, affect the mutual extensibility. Modulus of cross-links increase also very sharply near the percolation limit, the fact which cannot be understood if all cross-links were ascribed to the topological ties in the amorphous phase - as the crystallinity of material grows, and amorphous phase ceases out, no topological ties can explain the 15 times growth of $N_{c} \mathrm{kT}$. Let's also note that contribution of the cross-links is substantial starting only from intermediate stages of orientation. At low deformation all the links can be considered as slip-links, however as $N_{c} \ll N_{S}$, this additional contribution is negligibly small.

\section{Conclusion}

Studies of polypropylene with different degrees of isotacticity have shown a way of the rational design of material with predetermined mechanical properties starting from the synthesis stage already - controlled introducement of stereodefects will allow the smooth adjustment of the Young modulus and elasticity in the range from thermotropic to elastomer materials. It was also revealed that modern theoretical models of the elasticity can be successfully applied not only for the description of the mechanical behaviour of polymers, but also for better understanding of the mechanism of elasticity in them. While in the low crystalline materials deformation has Gaussian nature, in the materials of the intermediate crystallinity (30-40\%) percolation takes place, and the cross-linking net becomes more hard, manifesting the switch to the thermotropic behavior of the material. Simultaneously the divide between cross- and slip-links becomes substantial, as the parameter of extensibility grows sharply. 


\section{Acknowledgements}

This work was supported by Russian Science Foundation (project 14-1301402).

[1] N. R. Legge, G. Holden, H. E. Schroeder, Thermoplastic Elastomers, Hanser Publishers, 1996.

[2] J. G. Van Alsten, B. B. Sauer, C. R. Gochanour, K. L. Faron, W. D. Dozier, Interfacial Broadening and Self-Adhesion of Segmented Block Copolymers Studied by Neutron Reflection, Macromolecules 28 (20) (1995) 7019-7021. doi: 10.1021/ma00124a045.

[3] Yu. K. Godovsky, E. V. Konyukhova, S. N. Chvalun, V. M. Neverov, A. S. Abu-Surrah, B. Rieger, Stretching calorimetry and X-ray characterization of deformational behavior of new high molecular weight propene-carbon monoxide alternating co- and terpolymers, Macromol. Chem. Phys. 200 (1999) 2636-2644. doi: 10.1002/(SICI)15213935(19991201)200:12く2636::AID-MACP2636>3.0.CO;2-\#

[4] U. Dietrich, M. Hackmann, B. Rieger, M. Klinga, M. Leskelä, Control of Stereoerror Formation with High-Activity "Dual-Side" Zirconocene Catalysts: A Novel Strategy To Design the Properties of Thermoplastic Elastic Polypropenes, J. Am. Chem. Soc. 121 (1999) 4348-4355. doi: 10.1021/ja9833220.

[5] K. P. Gallagher, X. Zhang, J. P. Runt, G. Huynh-ba, J. S. Lin, Miscibility and cocrystallization in homopolymer-segmented block copolymer blends, Macromolecules 26 (4) (1993) 588-596. doi: 10.1021/ma00056a006.

[6] B. Rieger, C. Troll, J. Preuschen, Ultrahigh Molecular Weight Polypropene Elastomers by High Activity "Dual-Side" Hafnocene Catalysts, Macromolecules 35 (15) (2002) 5742-5743. doi: 10.1021/ma025520o.

[7] J. Voegele, C. Troll, B. Rieger, Zirconocene-catalyzed propene-ethene copolymer elastomers: Kinetic investigations at low ethene concentration and characterization of microstructure, Macromol. Chem. Phys.203 (13) (2002) 1918-1925. doi: 10.1002/1521-3935(200209)203:13<1918::AIDMACP1918>3.0.CO;2-4

[8] A. Schmidt, W. S. Veeman, V. M. Litvinov, W. Gabrielse, NMR Investigations of In-Situ Stretched Block Copolymers of Poly(butylene terephthalate) and Poly(tetramethylene oxide), Macromolecules 31 (5) (1998) 1652-1660. doi: 10.1021/ma9714676.

[9] H. H. Brintzinger, D. Fischer, R. Mülhaupt, B. Rieger, R. M. Waymouth, Stereospecific Olefin Polymerization with Chiral Metallocene Catalysts, Angew. Chem. Int. Ed. 34 (11) (1995) 1143-1170. doi: 10.1002/anie.199511431.

[10] L. Resconi, L. Cavallo, A. Fait, F. Piemontesi, Selectivity in Propene Polymerization with Metallocene Catalysts, Chem. Rev. 100 (4) (2000) 1253-1346. doi: 10.1021/cr9804691.

[11] G. W. Coates, Precise Control of Polyolefin Stereochemistry Using Single-Site Metal Catalysts, Chem. Rev. 100 (4) (2000) 1223-1252. doi: $10.1021 / \mathrm{cr} 990286 \mathrm{u}$. 
[12] S. Radhakrishnan, D. R. Saini, M. V. Kuber, Effect of Morphology on the dielectric properties of a segmented copolyester, Eur. Polym. J. 27 (3) (1991) 291-297. doi: 10.1016/0014-3057(91)90108-Z.

[13] E. J. Kramer, L. L. Berger, Fundamental processes of craze growth and fracture, Adv. Polym. Sci. 91/92 (1990) 1-68. doi: 10.1007/BFb0018018.

[14] A. M. Donald, E. J. Kramer, Effect of molecular entanglements on craze microstructure in glassy polymers, J. Polym. Sci. Phys. Ed. 20 (5) (1982) 899-909. doi: 10.1002/pol.1982.180200512.

[15] J. J. Mckechnie, R. N. Haward, D. Brown, J. H. R. Clarke, Effects of chain configurational properties on the stress-strain behavior of glassy linear polymers, Macromolecules 26 (1) (1993) 198-202. doi: 10.1021/ma00053a030.

[16] R. N. Haward, G. Thackray, The Use of a Mathematical Model to Describe Isothermal Stress-Strain Curves in Glassy Thermoplastics, Proc. R. Soc. Lond. A 302 (1968) 453-472. doi: 10.1098/rspa.1968.0029.

[17] M. C. Boyce, D. M. Parks, A. S. Argon, Large inelastic deformation of glassy polymers. Part II: numerical simulation of hydrostatic extrusion, Mech. Mater. 7 (1) (1988) 35-47. doi: 10.1016/0167-6636(88)90004-X.

[18] R. N. Haward, R. J. Young, The physics of glassy polymers, Chapman and Hall, 1997.

[19] L. R. G. Treloar, The physics of rubber elasticity, Oxford: Clarendon Press, 1975.

[20] Yu. K. Godovsky, Thermophysical methods of polymer studies, Moscow: Chemistry, 1976.

[21] D. T. Mallin, M. D. Rausch, Y. G. Lin, S. Dong, J. C. W. Chien, rac[Ethylidene(1-.eta.5-tetramethylcyclopentadienyl)(1-.eta.5-

indenyl)]dichlorotitanium and its homopolymerization of propylene to crystalline-amorphous block thermoplastic elastomers, J. Am. Chem. Soc. 112 (5) (1990) 2030-2031. doi: 10.1021/ja0016la075.

[22] G. W. Coates, R. M. Waymouth, Oscillating Stereocontrol: A Strategy for the Synthesis of Thermoplastic Elastomeric Polypropylene, Science 267 (1995) 217-219. doi: 10.1126/science.267.5195.217.

[23] U. Dietrich, M. Hackmann, B. Rieger, M. Klinga, M. Leskelä, Control of Stereoerror Formation with High-Activity "Dual-Side" Zirconocene Catalysts: A Novel Strategy To Design the Properties of Thermoplastic Elastic Polypropenes, J. Am. Chem. Soc. 121 (18) (1999) 4348-4355. doi: $10.1021 / j a 9833220$

[24] J. Kukral, P. Lehmus, T. Feifel, C. Troll, B. Rieger, Dual-Side ansaZirconocene Dichlorides for High Molecular Weight Isotactic Polypropene Elastomers, Organometallics 19 (19) (2000) 3767-3775. doi: 10.1021/om000224q.

[25] C. Cobzaru, S. Hild, A. Boger, C. Troll, B. Rieger, "Dual-side" catalysts for high and ultrahigh molecular weight homopolypropylene elastomers and plastomers, Coord. Chem. Rev. 250 (2006) 189-21l. doi: 10.1016/j.ccr.2005.06.007.

[26] A. Schöbel, D. Lanzinger, B. Rieger, Polymerization Behavior of ClSymmetric Metallocenes (M = Zr, Hf): From Ultrahigh Molecular Weight Elastic Polypropylene to Useful Macromonomers, Organometallics 32 (2) (2013) 427-437. doi: 10.1021/om30078la.

[27] A. Schöbel, E. Herdtweck, M. Parkinson, B. Rieger, Cover Picture: UltraRigid Metallocenes for Highly Iso- and Regiospecific Polymerization of Propene: The Search for the Perfect Polypropylene Helix, Chem.-Eur. J. 18 (14) (2012) 4129-4129. doi: 0.1002/chem.201290050. 
[28] M. R. Machat, D. Lanzinger, A. Pöthig, B. Rieger, Organometallics (2016). doi:10.1021/acs.organomet.6b00814.

[29] S. Brückner, S. V. Meille, V. Petraccone, B. Pirozzi, Polymorphism in isotactic polypropylene, Prog. Polym. Sci. 16 (2-3) (1991) 361-404. doi: 10.1016/0079-6700(91)90023-E.

[30] G. Natta, P. Corradini, Structure and properties of isotactic polypropylene, Nuovo Cimento 15 (1960) 40-51. doi: 10.1007/BF02731859.

[31] B. Lotz, S. Graff, S. Straupe, J. C. Wittmann, Single crystals of $\gamma$ phase isotactic polypropylene: combined diffraction and morphological support for a structure with non-parallel chains, Polymer 32 (16) (1991) 2902-2910. doi: 10.1016/0032-3861(91)90185-L.

[32] E. Lezak, Z. Bartezak, A. Galeski, Plastic Deformation of the $\gamma$ Phase in Isotactic Polypropylene in Plane-Strain Compression, Macromolecules 39 (14) (2006) 4811-4819. doi: 10.1021/ma0605907.

[33] C. de Rosa, F. Auriemma, O. R. de Ballesteros, D. de Luca, L. Resconi, The Double Role of Comonomers on the Crystallization Behavior of Isotactic Polypropylene: Propylene-Hexene Copolymers, Macromolecules 41 (6) (2008) 2172-2177. doi: 10.1021/ma071753+.

[34] E. Perez, D. Zucchi, M. C. Sacchi, F. Forlini, A. Bello, Obtaining the $\gamma$ phase in isotactic polypropylene: effect of catalyst system and crystallization conditions, Polymer 40 (3) (1999) 675-681. doi: 10.1016/S0032-3861(98)00291-2.

[35] C. de Rosa, F. Auriemma, M. Paolillo, L. Resconi, I. Camurati, Crystallization Behavior and Mechanical Properties of Regiodefective, Highly Stereoregular Isotactic Polypropylene: Effect of Regiodefects versus Stereodefects and Influence of the Molecular Mass, Macromolecules 38 (22) (2005) 9143-9154. doi: 10.1021/ma051004x.

[36] J. Arraz-Andres, B. Pena, R. Benavente, E. Perez, M. L. Cerrada, Influence of isotacticity and molecular weight on the properties of metallocenic isotactic polypropylene, Eur. Polym. J. 43 (6) (2007) 2357-2370. doi: 10.1016/j.eurpolymj.2007.03.034.

[37] V. I. Selikhova, N. P. Bessonova, E. V. Konyukhova, Ya. I. Odarchenko, E. A. Sinevich, S. N. Chvalun, B. Rieger, Effect of Stereoregularity on the Structure and Thermophysical Characteristics of Isotactic Polypropylene, Polym. Sci. Ser. A. 50 (10) (2008) 1071-1081. doi: 10.1134/S0965545X08100088.

[38] V. I. Selikhova, Ya. I. Odarchenko, N. P. Bessonova, E. A. Sinevich, M. A. Shcherbina, S. N. Chvalun, B. Rieger, Formation of the gamma phase in oriented isotactic polypropylene with varying stereoregularity, Polym. Sci. Ser. A. 57 (4) (2015) 404-414. doi: 10.1134/S0965545X15040136.

[39] B. Wunderlich, Macromolecular Physics, Academic Press, New York, 1980, V. 3, 61-64.

[40] U. Dietrich, M. Hackmann, B. Rieger, M. Klinga, M. Leskelä, Control of Stereoerror Formation with High-Activity "Dual-Side" Zirconocene Catalysts: A Novel Strategy To Design the Properties of Thermoplastic Elastic Polypropenes, J. Am. Chem. Soc. 121 (18) (1999) 4348-4355. doi: 10.1021/ja9833220.

[41] A. N. Ozerin, Yu. A. Zubov, V. I. Selikhova, S. N. Chvalun, N.F. Bakeev, Use of the method of measuring the absolute intensity of small angle $\mathrm{X}$ Ray scattering for the study of the structure of amorphous regions in oriented polyethylene films, Polymer Science U.S.S.R., 18 (9) (1976) 2434 2442. 
[42] A. N. Ozerin, Yu. A. Zubov, S.N. Chvalun, N. F. Bakeev, G. P. Belov, XRay structural analysis of amorphous regions in oriented films of linear polyethylene, Polymer Science U.S.S.R., 19 (5) (1977) 1223-1231.

[43] G. H. Vineyard. Patterson transforms and the interpretation of X-ray scattering from fibers. Acta Crystallogr. 4 (1951) 281.

[44] M. Rubinstein, R. H. Colby, Polymer Physics, Oxford University Press, 2003.

[45] A. Cohen, A Padé approximant to the inverse Langevin function, Rheol. Acta 30 (3) (1991) 270-273. doi: 10.1007/BF00366640.

[46] R. N. Haward, The application of non-Gaussian chain statistics to ultralow density polyethylenes and other thermoplastic elastomers, Polymer 40 (21) (1999) 5821-5832. doi: 10.1016/S0032-3861(99)00124-X.

[47] R. C. Ball, M. Doi, S. F. Edwards, M. Warner, Elasticity of entangled networks, Polymer 22 (8) (1981) 1010-1018. doi: 10.1016/00323861(81)90284-6.

[48] P. Thirion, T. Weil, Assessment of the sliding link model of chain entanglement in polymer networks, Polymer 25 (5) (1984) 609-614. doi: 10.1016/0032-3861(84)90027-2.

[49] S. F. Edwards, Th. Vilgis, The effect of entanglements in rubber elasticity, Polymer 27 (4) (1986) 483-492. doi: 10.1016/00323861(86)90231-4.

[50] A. Pawlak, A. Galeski, A. Rozanski, Cavitation during deformation of semicrystalline polymers, Prog. Polym. Sci. 39 (2014) 921-958. doi: 10.1016/j.progpolymsci.2013.10.007.

[51] M. G. Brereton, P. G. Klein, Analysis of the rubber elasticity of polyethylene networks based on the slip link model of S. F. Edwards et al., Polymer 29 (8) (1988) 970-974. doi: 10.1016/0032-3861(88)90002-X. 
Isotactic polypropylenes with different number of stereodefects were studied.

Haward and Doi and Edwards models were successfully applied.

Role of entanglements of different nature was elucidated.

A way to precise control the mechanical properties of polymers was proposed. 